Wien klin Mag 2014 · 17:1

DOI 10.1007/s00740-014-0015-4

Online publiziert: 30. August 2014

○) Springer-Verlag Wien 2014

Verena Kienast

Redakteur/Springer Medizin, Wien, Österreich

\title{
Mut zur Veränderung
}

Mit dem Beschluss der Gesundheitsreform auf politischer Ebene ist ein jahrelanges Projekt nun endlich entschieden worden. Die Weichen in eine, den neuen Entwicklungen angepasste, Zukunft sollen damit gestellt, das Gesundheitssystem und die Gesundheitsversorgung der Menschen hierzulande damit abgesichert werden. Es war ein langes Ringen und Verhandeln und in letzter Sekunde wurde auch noch die gleichberechtigte Stellung der an der Patientenbetreuung beteiligten Gesundheitsberufe gekippt und der Arzt als Koordinator erkoren. Die Ärztekammer hat also die Bedrohung, dass andere Berufsgruppen die Oberhoheit des Arztes untergraben könnten, erfolgreich abgewehrt. Vielleicht ja auch ein Machtspiel... Aber freilich ist die Zustimmung aller Beteiligten - und speziell der Gruppe der Ärzte - essentiell, um die Identifikation mit der Entscheidung zu erhöhen.

\section{Veränderungsresistenz und sinnentleerte Altlasten}

Jetzt wird es also an die Umsetzung des in seiner Ehrgeizigkeit schon im Vorfeld etwas beschnittenen Projekts gehen. Interdisziplinäre Praxen, neue Kooperationen aber auch die elektronische Datenarchivierung und -abrufbarkeit stehen nach wie vor zur Diskussion. Die Strukturen, die dafür notwendig sind, müssen teilweise erst geschaffen werden, bedürfen der Einbindung zahlreicher Akteure und machen das Arbeiten zu Beginn vielleicht nicht gleich unbedingt leichter. Neue Wege sind immer mit einem gewissen Risiko verbunden. Und der Mensch neigt im allgemeinen dazu, grundlegende Veränderungen zu scheuen. Langsame, schrittweise Modifikationen sind dem menschlichen Beharrungsvermögen zuträglicher. Lieb gewonnene Gewohnheiten gibt man nicht so gerne auf - auch wenn sinnentleerte Altlasten irgendwann zu beträchtli- chen Hindernissen und Blockaden in der täglichen Praxis kumulieren.

Mut zur Veränderung ist also angesagt. Mut zu Veränderungen in Strukturen, in Hierarchien und auch in den eigenen Sichtweisen. Vielleicht hilft bisweilen der Schritt zurück und ein bisschen Distanz - zu uns selbst und zum Geschehen um uns herum. Jener Ausschnitt der Welt, den wir aus unserer Perspektive wahrnehmen können, ist eben doch winzig und vielleicht nicht allgemeingültig. Die Sichtweise des Anderen einzunehmen, seine Motivation und Beweggründe, seine Ziele und seinen Weg dorthin zu sehen, erweitert auch die eigene Sicht der Welt, öffnet neue Möglichkeiten und verbessert das Verständnis. Eine Frage der Kommunikation.

\section{Ambitionierter Anspruch: Der ideale Sprechakt}

Für den Sozialphilosophen Jürgen Habermas bedarf es für eine tatsächlich gelungene Kommunikation einer idealen Sprechaktsituation, die allen Beteiligten die gleichen Chancen auf Beginn und Beteiligung am Dialog gibt sowie auf die Möglichkeit zur Deutung und Argumentation. Sie bedarf aber auch der Herrschaftsfreiheit und muss wahrhaftig sein. Natürlich handelt es sich hier um einen idealen Anspruch, der sich nicht für jede Kommunikationssituation anwenden lässt. Die Überprüfung der zahllosen alltäglichen Gesprächssituationen anhand der aufgestellten Maximalforderungen zeigt freilich ein eher tristes Bild: Hierarchien und Abhängigkeiten, aber auch unterschiedliche Informationsmöglichkeiten und nicht zuletzt die mangelnde Wahrhaftigkeit führen in der überwiegenden Zahl der Situationen $\mathrm{zu}$ einer reichlich verzerrten Kommunikation - im Berufsalltag, aber auch im Privatleben und in der öffentlichen Kommunikation. Aber vielleicht hilft es ja schon ein bisschen, sich diese Kriterien in manch offenbar unerquicklicher Kommunikation ins Gedächtnis zu rufen, um den entscheidenden Schwenk zur Verbesserung zu ermöglichen

Meint Ihre

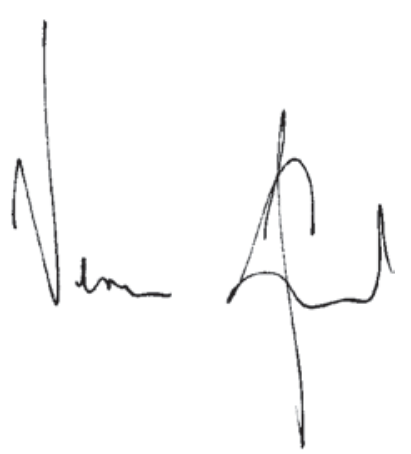

\section{Korrespondenzadresse}

\section{Kienast}

Redakteur/Springer Medizin Sachsenplatz 4-6, 1201 Wien verena.kienast@springer.at 